COMMUNICATIONS IN

NUMBER THEORY AND PHYSICS

Volume 8, Number 4, 703-727, 2014

\title{
Algebraic cycles and local quantum cohomology
}

\author{
Charles F. Doran and Matt Kerr
}

We review the Hodge theory of some classic examples from mirror symmetry, with an emphasis on what is intrinsic to the A-model. In particular, we illustrate the construction of a quantum $\mathbb{Z}$-local system on the cohomology of $K_{\mathbb{P}^{2}}$ and suggest how this should be related to the higher algebraic cycles studied in [10].

This note concerns three types of polarized variations of mixed Hodge structure (PVMHS) which arise in mirror symmetry:
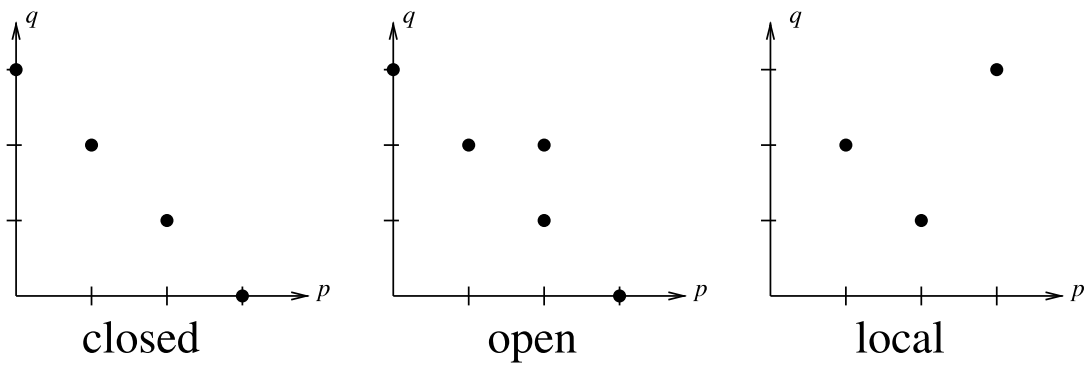

In each case, at the large complex structure boundary point one obtains a limiting mixed Hodge structure (LMHS) of Hodge-Tate type. It follows that replacing $W_{\bullet}$ by the relative weight filtration $M_{\bullet}$ produces a new PVMHS of the form
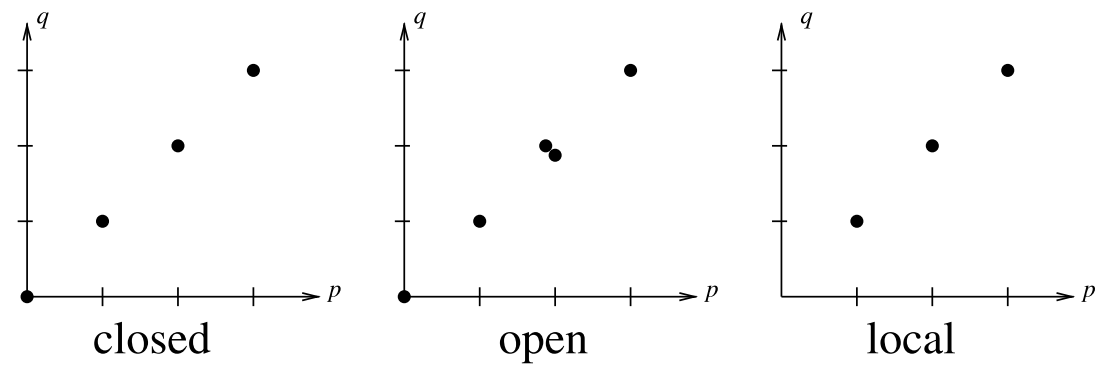

simultaneously in the A and B models. In particular, the $F^{p} \cap M_{2 p}$ subspaces identify with $H^{3-p, 3-p}$ in quantum cohomology. 
Let $\Delta^{*}$ denote the punctured unit disk and write $\mathcal{O}_{\Delta^{*}}=: \mathcal{O}, \Omega_{\Delta^{*}}^{1}=: \Omega^{1}$. A PVMHS $\left(\mathbb{V}, \mathcal{V}, \mathcal{F}^{\bullet}, W_{\bullet}, \nabla, Q\right)$ over $\Delta^{*}$ comprises

- a $\mathbb{Z}$-local system $\mathbb{V}$ on $\Delta^{*}$,

- the holomorphic vector bundle $\mathcal{V}$ with sheaf of sections $\mathbb{V} \otimes \mathcal{O}$,

- a decreasing filtration by holomorphic subbundles $\mathcal{F}^{j} \subset \mathcal{V}$,

- an increasing filtration by sublocal systems $W_{i} \subset \mathbb{V}_{\mathbb{Q}}:=\mathbb{V} \otimes \mathbb{Q}$,

- a flat connection $\nabla: \mathcal{V} \rightarrow \mathcal{V} \otimes \Omega^{1}$ with $\nabla\left(\mathcal{F}^{\bullet}\right) \subset \mathcal{F}^{\bullet-1}$ and $\nabla(\mathbb{V})=0$ and

- bilinear forms $Q_{i}:\left(\operatorname{Gr}_{i}^{W} \mathbb{V}\right)^{\otimes 2} \rightarrow \mathbb{Z}$,

such that each $\left(\mathrm{Gr}_{i}^{W} \mathbb{V}_{s}, \mathrm{Gr}_{i}^{W} \mathcal{F}_{s}^{\bullet}, Q_{i}\right)\left(s \in \Delta^{*}\right)$ yields a polarized Hodge structure. The PVMHS considered here, as well as all PVMHS arising from geometry, are admissible - i.e., have well-defined LMHS at 0.

In the above pictures, the number of bullets in position $(p, q)$ signifies the dimension of the summand in the Deligne bigrading on $\mathcal{V}$ defined pointwise by

$$
I^{p, q}\left(\mathcal{V}_{s}\right):=F^{p} \cap W_{p+q} \cap\left(\overline{F^{q}}+\sum_{j \geq 0}\left\{\overline{F^{q-j-1}} \cap W_{p+q-j-2}\right\}\right) .
$$

This bigrading is uniquely determined by the properties

(1) $\oplus_{p \geq j} \oplus_{q} I^{p, q}\left(\mathcal{V}_{s}\right)=\mathcal{F}_{s}^{j}$,

(2) $\oplus_{p+q \leq i} I^{p, q}\left(\mathcal{V}_{s}\right)=\left(W_{i}\right)_{s} \otimes \mathbb{C}$,

(3) $\overline{I^{b, a}\left(\mathcal{V}_{s}\right)} \equiv I^{a, b}\left(\mathcal{V}_{s}\right)$ modulo $\oplus_{p<a} \oplus_{q<b} I^{p, q}\left(\mathcal{V}_{s}\right)$.

In passing to the limit, heuristically one may visualize the bullets in each line $p+q=i$ moving up and down in such a way that the end result remains symmetric about this line.

Notation: Set $\ell(s):=\frac{\log (s)}{2 \pi i}$. We shall often write $\mathcal{V}$ (instead of the sixtuple) for a PVMHS.

\section{Closed string}

Beginning on the B-model side, recall how the LMHS construction works for a pure $\left(\mathbb{Z}_{-}\right)$VHS $\mathcal{V}$ of weight 3 over $\Delta^{*}$ with unimodular polarization 
$Q$. The weight filtration is the trivial one $W_{3}=\mathcal{V} \supset W_{2}=\{0\}$. Denote the (unipotent part of the) monodromy operator by $T$, with nilpotent logarithm

$$
N:=\log (T): \mathbb{V}_{\mathbb{Q}} \rightarrow \mathbb{V}_{\mathbb{Q}}
$$

There exists an unique filtration

$$
M_{-1}=\{0\} \subset M_{0} \subset M_{1} \subset \cdots \subset M_{6}=\mathbb{V}_{\mathbb{Q}}
$$

satisfying $N\left(M_{\alpha}\right) \subset M_{\alpha-2}$ and $N^{\ell}: \mathrm{Gr}_{3+\ell}^{M} \cong \mathrm{Gr}_{3-\ell}^{M}$. Untwisting the local system by

$$
\tilde{\mathbb{V}}:=e^{-\ell(s) N} \mathbb{V}
$$

we obtain the canonical extension

$$
\mathcal{V}_{e}:=\tilde{\mathbb{V}} \otimes \mathcal{O}_{\Delta}
$$

Let $\left\{\gamma_{i}\right\}$ be a multivalued basis of $\mathbb{V}$ generating the steps of the integral filtration $M_{m}^{\mathbb{Z}}:=\mathbb{V} \cap M_{m}$, and set $\tilde{\gamma}_{i}:=e^{-\ell(s) N} \gamma_{i} \in \Gamma(\Delta, \tilde{\mathbb{V}})$.

Definition 1.1. The LMHS of $\mathcal{V}$, denoted informally $V_{\text {lim }}$, is given by the data $V_{\lim }^{\mathbb{Z}}:=\mathbb{Z}\left\langle\left\{\tilde{\gamma}_{i}(0)\right\}\right\rangle, \mathcal{F}_{\lim }^{\bullet}:=\mathcal{F}_{e}^{p}(0)$ and (monodromy weight) filtration $M_{\bullet}$ on $V_{\lim }:=\mathcal{V}_{e}(0)$.

Assume that $V_{\text {lim }}$ is Hodge-Tate, i.e., $\operatorname{Gr}_{2 j}^{M} \cong \mathbb{Z}(-j)^{\oplus d_{j}}$ for $j=0,1,2,3$ and $\{0\}$ otherwise. (For example, the large complex structure LMHS for $H^{3}$ of the quintic mirror is of this type, whereas that for the Fermat quintic pencil $^{1}$ is not.) In the rank 4 setting, where we must have all $d_{j}=1$, we may pick (for each $j$ ) a holomorphic section $e_{j} \in \Gamma\left(\Delta, \mathcal{F}_{e}^{j} \cap M_{2 j}^{\mathbb{C}}\right.$ ) mapping to the image of $\gamma_{j} \in \Gamma\left(\mathfrak{H}, M_{2 j}^{\mathbb{Z}}\right)$ in $\Gamma\left(\Delta^{*}, \mathrm{Gr}_{2 j}^{M} \mathcal{V}\right)$ hence generating the latter. (Here $\mathfrak{H}$ denotes the upper half-plane.) Write $e=\left\{e_{3}, e_{2}, e_{1}, e_{0}\right\}$ and $\gamma=$ $\left\{\gamma_{3}, \gamma_{2}, \gamma_{1}, \gamma_{0}\right\}$ for the two bases.

\footnotetext{
${ }^{1}$ The $t=0$ LMHS $H_{\lim }^{3}\left(Y_{t}\right)$ of the pencil $Y_{t}:=\left\{t \sum_{i=0}^{4} X_{i}^{5}=\prod_{i=0}^{4} X_{i}\right\} \subset \mathbb{P}^{4}$ has nonzero $\operatorname{Gr}_{3}^{W}$ (cf. the analysis in [13, Section IV]).
} 
To make things explicit, we have (for some $a, b \in \mathbb{Z}$ and $e, f \in \mathbb{Q}$ )

$$
[Q]_{\gamma}=\left(\begin{array}{cccc}
0 & 0 & 0 & 1 \\
0 & 0 & 1 & 0 \\
0 & -1 & 0 & 0 \\
-1 & 0 & 0 & 0
\end{array}\right)=[Q]_{e} \text { and }[N]_{\gamma}=\left(\begin{array}{cccc}
0 & 0 & 0 & 0 \\
a & 0 & 0 & 0 \\
e & b & 0 & 0 \\
f & e & -a & 0
\end{array}\right)
$$

(cf. [12]), in which we shall demand that $|a|=1$. Replacing the local coordinate $s$ by $q:=e^{2 \pi \sqrt{-1} \tau}$, where $\tau:=Q\left(\gamma_{1}, e_{3}\right)$, and making full use of the bilinear relations (e.g., $Q\left(\mathcal{F}^{1}, \mathcal{F}^{3}\right)=0=Q\left(\mathcal{F}^{2}, \mathcal{F}^{2}\right)$ ), the limiting period matrix becomes (cf. [op. cit.])

$$
\tilde{\gamma}(0)[\mathbf{1}]_{e(0)}=\left(\begin{array}{cccc}
1 & 0 & 0 & 0 \\
0 & 1 & 0 & 0 \\
\frac{f}{2} & e & 1 & 0 \\
\alpha_{0} & \frac{f}{2} & 0 & 1
\end{array}\right)
$$

Example 1.2. For the mirror quintic family, we have (cf. [op. cit.], where the computation is based on [1]) $a=-1, b=5, e=\frac{11}{2}, f=-\frac{25}{6}$ and $\alpha_{0}=$ $\frac{25 i}{\pi^{3}} \zeta(3)=: C$.

Following Deligne [9], the $\left.e_{j}(q)\right|_{\Delta^{*}}$ provide the Hodge(-Tate) basis of a $\operatorname{PVMHS}\left(\mathbb{V}, \mathcal{V}, \mathcal{F}^{\bullet}, M_{\bullet}, \nabla\right)$ on $\Delta^{*}$, denoted $\mathcal{V}_{\text {rel }}$ for short. For the connection, we have

$$
[\nabla]_{e}=d+\left(\begin{array}{cccc}
0 & 0 & 0 & 0 \\
1 & 0 & 0 & 0 \\
0 & -Y(q) & 0 & 0 \\
0 & 0 & -1 & 0
\end{array}\right) \otimes \frac{d q}{(2 \pi \sqrt{-1}) q}
$$

where $Y(q)$ defines the Yukawa coupling. In the event that $\mathcal{V}$ comes from $H^{3}(X)$, and $\Phi$ denotes the Gromov-Witten prepotential of the mirror $X^{\circ}$ (composed with the inverse mirror map), according to mirror symmetry we have $Y=\Phi^{\prime \prime \prime}:=\frac{d^{3} \Phi}{d \tau^{3}}$.

Example 1.3. The mirror quintic VHS arises from $H^{3}$ of $X_{\xi}$, which is a smooth compactification of

$$
\left\{1-\xi\left(\sum_{i=1}^{4} x_{i}+\frac{1}{\prod_{i=1}^{4} x_{i}}\right)=0\right\} \subset\left(\mathbb{C}^{*}\right)^{\times 4}
$$


Taking $s:=\xi^{5}$, we obtain $\tau$ and $q$ as above, and

$$
\Phi(q)=\frac{5}{6} \tau^{3}+\Phi_{h}(q)
$$

where the holomorphic part

$$
\Phi_{h}(q)=\frac{1}{(2 \pi i)^{3}} \sum_{d \geq 1} N_{d} q^{d} .
$$

From $[1,12,23]$, we have the mixed Hodge basis

$$
\begin{aligned}
e_{0}= & \gamma_{0}, \\
e_{1}= & \gamma_{1}-\tau \gamma_{0}, \\
e_{2}= & \gamma_{2}-\left(5 \tau+\frac{11}{2}+\Phi_{h}^{\prime \prime}\right) \gamma_{1}+\left(\frac{5}{2} \tau^{2}+\frac{25}{12}+\tau \Phi_{h}^{\prime \prime}-\Phi_{h}^{\prime}\right) \gamma_{0}, \\
e_{3}= & \gamma_{3}+\tau \gamma_{2}-\left(\frac{5}{2} \tau^{2}+\frac{11}{2} \tau-\frac{25}{12}+\Phi_{h}^{\prime}\right) \gamma_{1} \\
& +\left(\frac{5}{6} \tau^{3}+\frac{25}{12} \tau-C+\tau \Phi_{h}^{\prime}-2 \Phi_{h}\right) \gamma_{0} .
\end{aligned}
$$

Here $e_{3}$ can also be viewed as the class of a holomorphic 3 -form in the original VHS, whose LMHS is reflected by the presence of $C$. The mirror $X^{\circ}$ is the Fermat quintic.

Turning to the A-model, we need to define an integral structure, Hodge and weight filtrations on

$$
H^{\text {even }}\left(X^{\circ}\right)=H^{3,3} \oplus H^{2,2} \oplus H^{1,1} \oplus H^{0,0},
$$

which will lead to VHS, LMHS and VMHS isomorphic to those on $H^{3}(X)$. These variations will be defined over a small disk $0<|q|<\epsilon$. For constructing them, the general idea is to use the family of algebraic structures on $H^{\text {even }}$ parametrized by $\tau[H] \in H^{1,1}\left(X^{\circ}\right)$, known as the (small) quantum cohomology. (Here $[H]$ the the class of a hyperplane section and $\tau=\ell(q)$, and we are working in the rank 4 setting.)

For the filtrations, we set

$$
F^{a} H^{\text {even }}=\oplus_{i \leq 3-a} H^{i, i}, \quad M_{b} H^{\text {even }}=\oplus_{j \geq 3-\frac{b}{2}} H^{j, j},
$$

so that $\mathcal{F}^{3-k} \cap M_{6-2 k}=H^{i, i}\left(X^{\circ}, \mathbb{C}\right)$ as a subspace of $H^{\text {even }}$. This is where the "naive" fundamental classes of coherent sheaves or algebraic cycles of 
codimension $i$ lie. In contrast, the integral local system will be generated by quantum-deformed fundamental classes of algebraic cycles on $X^{\circ}$. Alternately, we can regard the flat structure as given by the solution to a quantum differential equation

$$
\nabla=d+E \otimes \frac{d q}{(2 \pi \sqrt{-1}) q},
$$

which gives the integral structure up to a constant. (Note that $d$ differentiates with respect to $\oplus_{i} H^{i, i}\left(X^{\circ}, \mathbb{C}\right) ; E$ is an $\mathcal{O}\left(\Delta^{*}\right)$-linear operator we shall illustrate in the example below.) Since $E$ kills $M$-graded pieces, we get a natural identification between $\mathrm{Gr}_{2 i}^{M}$ of this "integral structure" and $H^{i, i}\left(X^{\circ}, \mathbb{Z}\right)$.

Example 1.4. For $X^{\circ}$ the Fermat quintic, we have Hodge basis

$$
\left[X^{\circ}\right]=e_{3}, \quad[H]=e_{2}, \quad-[L]=e_{1}, \quad[p]=e_{0},
$$

where $H$ is a hyperplane section, $L$ a line and $p$ a point. The minus sign on $[L]$ ensures that the form

$$
Q(\alpha, \beta):=(-1)^{\frac{\operatorname{deg}(\alpha)}{2}} \int_{X^{\circ}} \alpha \cup \beta
$$

has matrix $[Q]_{e}$ as above, which is necessary for equality of polarized VHS.

For the quantum deformed classes, we invert the relations of Example 1.3 to obtain

$$
\begin{aligned}
{\left[X^{\circ}\right]_{\mathcal{Q}}=} & \gamma_{3}=\left[X^{\circ}\right]-\tau[H]+\left(\frac{5}{2} \tau^{2}+\frac{25}{12}+\tau \Phi_{h}^{\prime \prime}-\Phi_{h}^{\prime}\right)[L] \\
& +\left(-\frac{5}{6} \tau^{3}-\frac{25}{12} \tau+C-\tau \Phi_{h}^{\prime}+2 \Phi_{h}\right)[p] \\
{[H]_{\mathcal{Q}}=} & \gamma_{2}=[H]-\left(5 \tau+\frac{11}{2}+\Phi_{h}^{\prime \prime}\right)[L]+\left(\frac{5}{2} \tau^{2}+\frac{11}{2} \tau-\frac{25}{12}+\Phi_{h}^{\prime}\right)[p] \\
{[L]_{\mathcal{Q}}=} & -\gamma_{1}=[L]-\tau[p], \\
{[p]_{\mathcal{Q}}=} & \gamma_{0}=[p] .
\end{aligned}
$$

These are solutions to the above differential equation with $E$ given by the (small) quantum product $[H] *$ defined by

$$
[H] *\left[X^{\circ}\right]=[H], \quad[H] *[H]=\Phi^{\prime \prime \prime}[L], \quad[H] *[L]=[p], \text { and }[H] *[p]=0 .
$$


(Note that this is consistent with cup product, in the sense that $[H] \cup[H]=$ $\left.5[L]=\Phi^{\prime \prime \prime}(0)[L].\right)$ The resulting variations of $\mathrm{HS}$ on $H^{\text {even }}\left(X^{\circ}\right)$ and $H^{3}(X)$ match by construction.

The natural question at this point is: how much of this "common $\mathbb{Z}$ VHS" is intrinsic to the A-model, and not just the B-model? Clearly the issue lies not in the Hodge and monodromy weight filtrations (given by the grading of $H^{\text {even }}$ by degree), or the polarizing form $Q$, or the $\nabla$-flat complex local system (given by the quantum product), but in the integral structure on the latter. Another way to think of this (cf. [9]) is that we must determine the "constant of integration" of the VHS, or equivalently the LMHS (1.2).

Naively, one could try to find a basis $\delta$ of the local system with integral $[Q]_{\delta}$ and integral monodromy matrices (which are computable in principle by analytic continuation). Unfortunately the result may not be unique, even after identifying bases related by a rational symplectic matrix. In the above example, one could have

$$
\delta_{3}=\frac{\gamma_{3}}{\sqrt{5}}+\frac{\gamma_{2}}{\sqrt{5}}, \quad \delta_{2}=\frac{\gamma_{2}}{\sqrt{5}}-\frac{3 \gamma_{1}}{\sqrt{5}}-\frac{3 \gamma_{0}}{\sqrt{5}}, \quad \delta_{1}=\sqrt{5} \xi_{1}, \quad \delta_{0}=\sqrt{5} \gamma_{0}
$$

which produces the (distinct) quintic twin mirror $\mathbb{Z}$-VHS. Indeed, in [11] this phenomenon is responsible for the bifurcation of each $\mathbb{R}-\mathrm{VHS}$ into finitely many distinct $\mathbb{Z}$-VHS.

Instead, what is needed is a direct construction of an integral structure on quantum cohomology, which has only recently been realized by Iritani $[15,16]$ and Katzarkov-Kontsevich-Pantev [18]. We illustrate how this works in the setting where $X^{\circ}$ is a smooth CY 3-fold, and $\operatorname{dim} H^{\text {even }}\left(X^{\circ}\right)=4$. A map $\sigma$ from $H^{\text {even }}$ to multivalued $\nabla$-flat sections (in a neighborhood of $q=0$ ), defined in terms of Gromov-Witten theory, has been known for some time (cf. [7, Sections 8.5.3, 10.2.2]). If $\alpha_{i} \in H^{2(3-i)}\left(X^{\circ}\right)(i=0,1,2,3)$ denote a $Q$-symplectic basis with $\alpha_{2}=[H]$, this boils down to first setting

$$
\begin{aligned}
& \tilde{\sigma}\left(\alpha_{0}\right):=\alpha_{0}, \quad \tilde{\sigma}\left(\alpha_{1}\right):=\alpha_{1}, \quad \tilde{\sigma}\left(\alpha_{2}\right):=\alpha_{2}+\Phi_{h}^{\prime \prime} \alpha_{1}+\Phi_{h}^{\prime} \alpha_{0}, \\
& \tilde{\sigma}\left(\alpha_{3}\right):=\alpha_{3}+\Phi_{h}^{\prime} \alpha_{1}+2 \Phi_{h} \alpha_{0}
\end{aligned}
$$

and then

$$
\sigma(\alpha):=\tilde{\sigma}\left(e^{-\tau[H]} \cup \alpha\right):=\sum_{k \geq 0} \frac{(-1)^{k}}{k !} \tilde{\sigma}\left([H]^{k} \cup \alpha\right)
$$


(In our running example, we obviously have in mind $\alpha_{3}=\left[X^{\circ}\right], \alpha_{2}=[H]$, $\alpha_{1}=-[L]$, and $\alpha_{0}=[p]$.) These are $\nabla$-flat sections with monodromy

$$
T(\sigma(\alpha))=\sigma\left(e^{-[H]} \cup \alpha\right) .
$$

We also set $\sigma_{\infty}(\alpha):=\left.\tilde{\sigma}(\alpha)\right|_{q=0}$.

The key new ingredient introduced by the authors $[15,18]$ is a characteristic class defined using the $\Gamma$-function, and which in our setting specializes to

$$
\hat{\Gamma}\left(X^{\circ}\right):=\exp \left(\sum_{k \geq 2} \frac{(-1)^{k}(k-1) !}{(2 \pi i)^{k}} \zeta(k) \operatorname{ch}_{k}\left(T X^{\circ}\right)\right) \in H^{\text {even }}\left(X^{\circ}\right) .
$$

Using it, we may assign a flat section

$$
\gamma(\xi):=\sigma\left(\hat{\Gamma}\left(X^{\circ}\right) \cup \operatorname{ch}(\xi)\right)
$$

to each $\xi \in K_{0}^{\text {num }}\left(X^{\circ}\right)$, which defines a $\mathbb{Z}$-local system. (Similarly, we can define $\tilde{\gamma}(\xi), \gamma_{\infty}(\xi)$ by applying $\tilde{\sigma}, \sigma_{\infty}$.) A strong indication that $\hat{\Gamma}$ gives the right "correction" is Iritani's result (cf. [15, Proposition 2.10]) that the Mukai pairing

$$
\left\langle\xi, \xi^{\prime}\right\rangle:=\int_{X^{\circ}} \operatorname{ch}\left(\xi^{\vee} \otimes \xi^{\prime}\right) \cup T d\left(X^{\circ}\right)=Q\left(\gamma(\xi), \gamma\left(\xi^{\prime}\right)\right) .
$$

Moreover, since $\operatorname{ch}(\mathcal{O}(-1))=e^{-[H]},(1.3)$ implies that

$$
T(\gamma(\xi))=\gamma(\mathcal{O}(-1) \otimes \xi)
$$

- an elementary example of how a categorical autoequivalence of $D^{b}\left(X^{\circ}\right)$ corresponds to monodromy. The autoequivalences corresponding to monodromies arising away from $q=0$ have been explicitly identified in [5].

Example 1.5. Once more we take $X^{\circ}$ to be the Fermat quintic, which has total Chern class $c\left(X^{\circ}\right)=1+50[L]-200[p]$ and Todd class $T d\left(X^{\circ}\right)=$ $1+\frac{25}{6}[L]$. A Mukai-symplectic basis of $K_{0}^{\text {num }}\left(X^{\circ}\right)$ is

$$
\begin{aligned}
& \xi_{3}:=\mathcal{O}_{X^{\circ}}, \quad \xi_{2}:=\mathcal{O}_{H}-3 \mathcal{O}_{L}-8 \mathcal{O}_{p}, \quad \xi_{1}:=-\mathcal{O}_{L}-\mathcal{O}_{p} \equiv-\mathcal{O}_{L}(1), \\
& \xi_{0}:=\mathcal{O}_{p} ;
\end{aligned}
$$

this in fact (referring to Example 1.2 and (1.1)) satisfies $[\mathcal{O}(-1) \otimes]_{\xi}=$ $\exp \left([N]_{\gamma}\right)$. (Note that taking $\xi_{2}=\mathcal{O}_{H}$ and $\xi_{1}=\mathcal{O}_{L}$ does not yield a 
symplectic basis.) From

$$
\begin{aligned}
& \operatorname{ch}\left(\xi_{3}\right)=\left[X^{\circ}\right], \quad \operatorname{ch}\left(\xi_{2}\right)=[H]-\frac{11}{2}[L]-\frac{25}{6}[p], \quad \operatorname{ch}\left(\xi_{1}\right)=-[L], \\
& \operatorname{ch}\left(\xi_{0}\right)=[p]
\end{aligned}
$$

and $\hat{\Gamma}\left(X^{\circ}\right)=\left[X^{\circ}\right]+\frac{25}{12}[L]+C[p]$, a straightforward computation gives that

$$
\gamma\left(\xi_{i}\right)=\gamma_{i} \quad(i=0,1,2,3)
$$

with the $\left\{\gamma_{i}\right\}$ exactly as in Example 1.4. Moreover, the $\left\{\gamma_{\infty}\left(\xi_{i}\right)\right\}$ recover the LMHS matrix (1.2) (with $e, f, \alpha_{0}$ as in Example 1.2), including the crucial constant $C$ which visibly comes from $\hat{\Gamma}$.

Remark 1.6. The toric-hypersurface CY 3-fold families from which Bmodel VHS's are often produced are intrinsically defined over $\mathbb{Q}$. Moreover, by virtue of its toric nature, the large complex structure limit may be regarded as a $\mathbb{Q}$-semistable degeneration. The general conjectural framework surrounding the limiting motive (cf. [12, (III.B.5)]) therefore predicts that the class $\alpha_{0} \in \operatorname{Ext}_{\mathrm{MHS}}^{1}(\mathbb{Q}(-3), \mathbb{Q}(0)) \cong \mathbb{C} / \mathbb{Q}$ arising in the corresponding LMHS is always a rational multiple of the constant $\frac{\zeta(3)}{(2 \pi i)^{3}}$, motivating its appearance in (1.4). ${ }^{2}$

The "non-toric" degenerations at the conifold and Gepner points, on the other hand, produce singular fibers whose desingularization may introduce an algebraic extension of $\mathbb{Q}$, leading to an arithmetically richer LMHS. One should try to use mirror symmetry to get at this, perhaps beginning with

Problem 1.7. Adapt the (A-model) $\hat{\Gamma}$-integral structure on FJRW theory introduced in [5] to the explicit computation of the periods of (B-model) LMHS at the Gepner point $(s=\infty)$.

Remark 1.8. The periods of an LMHS are the entries of a matrix (such as (1.2)) relating rational (or integral) and Hodge bases, which are chosen compatibly with the monodromy weight filtration. ${ }^{3}$ Of greatest interest in Problem 1.7 (at least, when the monodromy about the Gepner point is not finite) is the explicit computation of nontorsion extension classes between

\footnotetext{
${ }^{2}$ Note that we are interested in the arithmetic of locally complete CY families; taking irrational "slices" of such to force an extension both misses the point and will not affect $\alpha_{0}$.

${ }^{3}$ For instance, one can take the $\left\{e_{i}(0)\right\}$ to give a basis of the Deligne bigrading of $\left(F_{\lim }^{\bullet}, M_{\bullet}\right)$.
} 
the $\left\{\mathrm{Gr}_{k}^{M}\right\}$ - possibly in terms of Gamma- and zeta-values, hypergeometric special values and the like. While Section 4 (also see Corollary 1.4) of [op. cit.] does not carry this out, it appears to contain the technical background for doing so.

See Section 4 for another source of algebraic extensions.

\section{Local string}

This section is based on a simple example studied by $[8,10,14,21]$. Once and for all we set

$$
Y_{\xi}:=\left\{(x, y ; u, v) \in\left(\mathbb{C}^{*}\right)^{2} \times \mathbb{C}^{2} \mid 1-\xi\left(x+y+\frac{1}{x y}\right)+u^{2}+v^{2}=0\right\},
$$

the so-called Hori-Vafa mirror of $Y^{\circ}=K_{\mathbb{P}^{2}}$. The canonical holomorphic $(3,0)$ form on $Y_{\xi}$ is given by

$$
\eta_{\xi}=2 \sqrt{-1} \operatorname{Res}_{Y_{\xi}}\left(\frac{\frac{d x}{x} \wedge \frac{d y}{y} \wedge d u \wedge d v}{1-\xi\left(x+y+\frac{1}{x y}\right)+u^{2}+v^{2}}\right) .
$$

The 3-cycles are spanned in homology by (a) a real 3-torus $\mathbb{T}^{3}$ and (b) circlebundles over membranes in $\left(\mathbb{C}^{*}\right)^{2}$ bounding 1-cycles on the thrice-punctured elliptic curve $W_{\xi}^{*}:=\left\{(x, y) \in\left(\mathbb{C}^{*}\right)^{2} \mid 1-\xi\left(x+y+\frac{1}{x y}\right)=0\right\}$. The circle is pinched to a point over the 1-cycles.

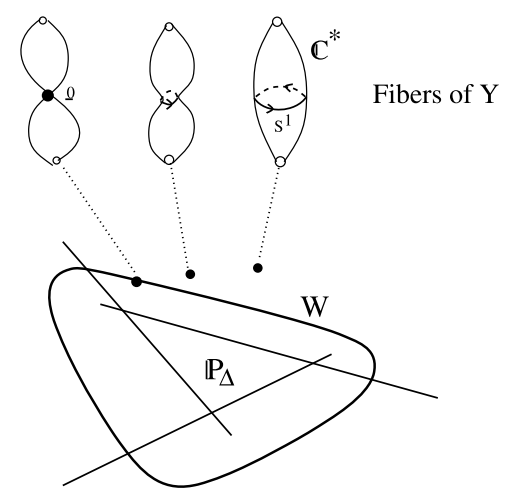

We write $W_{\xi}$ for the complete elliptic curve, $\tilde{\omega}_{\xi}:=\frac{1}{2 \pi i} \operatorname{Res}_{W_{\xi}}\left(\frac{\frac{d x}{x} \wedge \frac{d y}{y}}{1-\xi\left(x+y+\frac{1}{x y}\right)}\right)$ for the canonical holomorphic 1-form, and $\varphi_{0}, \varphi_{1}$ for 1-cycles spanning $H_{1}$ 
$\left(W_{\xi}, \mathbb{Z}\right)$ with periods $\pi_{i}:=\int_{\varphi_{i}} \tilde{\omega}_{\xi}$. In particular, we let $\varphi_{0}$ be the vanishing cycle and $\omega_{\xi}:=\tilde{\omega}_{\xi} / \pi_{0}$ the normalization of the 1 -form so that $\int_{\varphi_{0}} \omega_{\xi} \equiv 1$.

Denoting the membrane construction (b) by $\mathcal{M}$, we have the short exact sequence

$$
\begin{gathered}
0 \longrightarrow \mathbb{Z}\left\langle\mathbb{T}^{3}\right\rangle \longrightarrow H_{3}(Y) \longrightarrow \operatorname{ker}\left\{H_{1}\left(W^{*}\right) \rightarrow H_{1}\left(\left(\mathbb{C}^{*}\right)^{2}\right)\right\}(1) \longrightarrow 0 \\
\qquad \begin{array}{c}
\downarrow \\
H_{1}(W)(1)
\end{array}
\end{gathered}
$$

(cf. [DK, Section 5]). ${ }^{4}$ Its dual

$$
0 \longleftarrow \mathbb{Z}(-3) \longleftarrow H^{3}(Y) \longleftarrow \mu H^{1}(W)(-1) \longleftarrow 0
$$

yields an extension class

$$
\varepsilon \in \operatorname{Ext}_{\mathrm{MHS}}^{1}\left(\mathbb{Z}(-2), H^{1}(W)\right) \cong \operatorname{Hom}\left(H_{1}(W), \mathbb{C} / \mathbb{Z}(2)\right) .
$$

Miraculously, this is the image of a higher cycle $\Xi \in K_{2}^{\mathrm{alg}}(W)$ by a generalized Abel-Jacobi map [10], and the periods of $\eta$ may be described by

$$
\frac{1}{2 \pi \sqrt{-1}} \int_{\mathcal{M}(\gamma)} \eta \underset{\mathbb{Z}(2)}{\equiv}\langle A J(\Xi), \gamma\rangle_{W}, \quad \frac{1}{(2 \pi \sqrt{-1})^{3}} \int_{\mathbb{T}^{3}} \eta=1 .
$$

Normalizing the local coordinate $s:=\xi^{3}$ to $q$ where

$$
\ell(q):=\tau:=\frac{\pi_{1}}{\pi_{0}}=\int_{\varphi_{1}} \omega_{\xi},
$$

we remark that $s \mapsto q$ gives the mirror map for the family $W$ of elliptic curves. Similarly, if we set

$$
\ell(Q):=\mathcal{T}:=\frac{1}{(2 \pi \sqrt{-1})^{3}} \int_{\mathcal{M}\left(3 \varphi_{0}\right)} \eta,
$$

then $s \mapsto Q$ is the local mirror map for $Y$. The initial VMHS $\mathcal{V}$ is that on $H^{3}(Y)$, with integral basis $^{5} \gamma=\left\{\gamma_{3}, \gamma_{2}, \gamma_{1}\right\}$ where

$$
\gamma_{3}:=\mathbb{T}^{\vee}, \quad \gamma_{2}=\mathcal{M}\left(3 \varphi_{0}\right)^{\vee}, \quad \gamma_{1}=\mathcal{M}\left(3 \varphi_{1}\right)^{\vee}
$$

\footnotetext{
${ }^{4}$ The isomorphism is valid only rationally, but can be made integral by replacing $H_{1}(W, \mathbb{Z})$ by $\mathbb{Z}\left\langle 3 \varphi_{0}, \varphi_{1}\right\rangle$, which is done tacitly below.

${ }^{5}$ We will ignore for now the fact that $\gamma_{1}$ is really $\frac{1}{3}$ of an integral class; it is a more convenient choice for our purposes than $\mathcal{M}\left(\varphi_{1}\right)^{v}$.
} 
From the exact sequence we can read off the weight filtration

$$
W_{6}=\mathcal{V} \supset W_{5}=W_{4}=W_{3}=\left\langle\gamma_{2}, \gamma_{1}\right\rangle=\operatorname{im}\{\mu\} \supset W_{2}=\{0\},
$$

and Hodge filtration (except for $\mathcal{F}^{3}=\langle\eta\rangle$ ). The extension data are recorded by $\mathcal{T}=\left\langle A J(\Xi), 3 \varphi_{0}\right\rangle$ and $\Phi:=\left\langle A J(\Xi), 3 \varphi_{1}\right\rangle$.

The monodromy logarithm

$$
[N]_{\gamma}=\left(\begin{array}{ccc}
0 & 0 & 0 \\
-1 & 0 & 0 \\
\frac{1}{2} & -1 & 0
\end{array}\right)
$$

leads to a relative weight filtration $M_{\bullet}$. The resulting $\mathcal{V}_{\text {rel }}$ has Hodge-Tate basis

$$
\begin{aligned}
e_{3} & :=\frac{\eta}{(2 \pi \sqrt{-1})^{3}}=\gamma_{3}+\mathcal{T} \gamma_{2}+\Phi \gamma_{1} \in \mathcal{F}^{3} \cap M_{6}, \\
e_{2} & :=\mu(\omega)=\gamma_{2}+\tau \gamma_{1} \in \mathcal{F}^{2} \cap M_{4}, \\
e_{1} & =\gamma_{1} \in \mathcal{F}^{1} \cap M_{2} .
\end{aligned}
$$

From transversality

$$
\gamma_{2}+\frac{d \Phi}{d \mathcal{T}} \gamma_{1}=\nabla_{\partial_{\mathcal{T}}} e_{3} \in \mathcal{F}^{2}
$$

we deduce that $\frac{d \Phi}{d \mathcal{T}}=\tau$, which may also be derived from the fact that logarithmic derivatives of the extension classes give periods ${ }^{6}$ of $\tilde{\omega}_{\xi}$ [op. cit.]:

$$
\frac{d \Phi}{d \mathcal{T}}=\frac{s \cdot d \Phi / d s}{s \cdot d \mathcal{T} / d s}=\frac{\pi_{1}}{\pi_{0}}=\tau
$$

This equality has the important consequence

$$
\Phi^{\prime \prime}:=\frac{d^{2} \Phi}{d \mathcal{T}^{2}}=\frac{d \tau}{d \mathcal{T}}=\frac{\delta_{s}\left(\pi_{1} / \pi_{0}\right)}{\delta_{s} \mathcal{T}}=\frac{2 \pi \sqrt{-1}\left(\pi_{0} \delta_{s} \pi_{1}-\pi_{1} \delta_{s} \pi_{0}\right)}{\pi_{0}^{3}}=\frac{\mathcal{Y}}{\pi_{0}^{3}},
$$

where $\mathcal{Y}$ is the (suitably normalized) Yukawa coupling for the family $\left\{W_{\xi}\right\}$ of elliptic curves. Noting as well that $\nabla_{\partial_{\mathcal{T}}} e_{2}=\frac{d \tau}{d \mathcal{T}} e_{1}$, we conclude that

$$
[\nabla]_{e}=d+\left(\begin{array}{ccc}
0 & 0 & 0 \\
1 & 0 & 0 \\
0 & \Phi^{\prime \prime} & 0
\end{array}\right) \otimes \frac{d Q}{(2 \pi \sqrt{-1}) Q}
$$

where $e=\left\{e_{3}, e_{2}, e_{1}\right\}$.

\footnotetext{
${ }^{6}$ That is, we have $\delta_{s} \mathcal{T}=\frac{1}{2 \pi i} \pi_{0}, \delta_{s} \Phi=\frac{1}{2 \pi i} \pi_{1}$.
} 
Turning to the A-model, we shall seek a quantum interpretation of $\nabla$. Before doing so, we remark that by [14] and [10], under the local mirror map $\Phi$ may be identified as the local Gromov-Witten prepotential

$$
\Phi \equiv \frac{1}{2} \mathcal{T}^{2}-\frac{1}{(2 \pi \sqrt{-1})^{2}} \sum_{d} 3 d N_{d} Q^{d}
$$

modulo lower-order terms in $\mathcal{T} .{ }^{7}$ Differentiating (2.2) twice, we have

$$
1-\sum_{d} 3 d^{3} N_{d} Q^{d}=\frac{\mathcal{Y}}{\pi_{0}^{3}}
$$

in which the right-hand side has a pole where the family $W$ degenerates. Directly computing $\left\langle A J(\Xi), \varphi_{0}\right\rangle$ at this singular elliptic curve gives $\Im\left(\mathcal{T}_{0}\right)=$ $\frac{27 \sqrt{3}}{8 \pi^{2}} L\left(\chi_{-3}, 2\right)[10]$, and hence $Q_{0}=\left|e^{2 \pi \sqrt{-1} \mathcal{T}_{0}}\right|=e^{-2 \pi \Im\left(\mathcal{T}_{0}\right)}$ for the radius of convergence. This ties the asymptotic growth rate

$$
\limsup _{d \rightarrow \infty}\left|N_{d}\right|^{\frac{1}{d}}=e^{2 \pi \Im\left(\mathcal{T}_{0}\right)}
$$

of the local Gromov-Witten numbers directly to the Beilinson regulator of an algebraic cycle.

For the quantum interpretation, we consider the dual VMHS $\mathcal{V}^{\vee}$ on $H_{3}(Y)$ under the pairing $H^{3}(Y) \times H_{3}(Y) \rightarrow H_{0}(Y)=\mathbb{Z}$. The dual integral (flat) basis is of course

$$
\gamma_{1}^{\vee}=\mathbb{T}^{3}, \quad \gamma_{2}^{\vee}=\mathcal{M}\left(3 \varphi_{0}\right), \quad \gamma_{1}^{\vee}=\mathcal{M}\left(3 \varphi_{1}\right),
$$

and in the dual Hodge basis $e^{\vee}=\left\{e_{3}^{\vee}, e_{2}^{\vee}, e_{1}^{\vee}\right\}$ we have

$$
[\nabla]_{e^{\vee}}=d-\left(\begin{array}{ccc}
0 & 1 & 0 \\
0 & 0 & \Phi^{\prime \prime} \\
0 & 0 & 0
\end{array}\right) \otimes \frac{d Q}{2 \pi \sqrt{-1} Q}
$$

Now recalling that $Y^{\circ}=K_{\mathbb{P}^{2}}$, Hosono [14] proposed a homological mirror map

$$
\operatorname{mir}: K_{0}^{c}\left(Y^{\circ}\right) \rightarrow H_{3}(Y, \mathbb{Z})
$$

${ }^{7} \mathrm{~A}$ different form of this result is already present in Section 6.2 of [8], about which we shall say more in the next section. 
from coherent sheaves with compact support to homology classes of Lagrangian 3-cycles, given explicitly by

$$
\mathcal{O}_{p} \mapsto \gamma_{3}^{\vee}, \quad \mathcal{O}_{\mathbb{P}^{1}}(-1) \mapsto \gamma_{2}^{\vee}, \quad \mathcal{O}_{\mathbb{P}^{2}}(-2) \mapsto \gamma_{1}^{\vee}
$$

(The sheaves are all supported on the zero-section $\mathbb{P}^{2} \subset Y^{\circ}$.) Making the identifications $e_{3}^{\vee}=[p], e_{2}^{\vee}=\left[\mathbb{P}^{1}\right], e_{1}^{\vee}=\left[\mathbb{P}^{2}\right]$ under $\overline{\operatorname{mir}}: H_{\text {even }}\left(Y^{\circ}\right) \stackrel{\cong}{\rightrightarrows} H_{3}$ $(Y)$, we impose as before an integral structure on the A-model side by means of the quantum deformed classes

$$
([p]=)[p]_{\mathcal{Q}}:=\gamma_{3}^{\vee}, \quad\left[\mathbb{P}^{1}\right]_{\mathcal{Q}}:=\gamma_{2}^{\vee}, \quad\left[\mathbb{P}^{2}\right]_{\mathcal{Q}}:=\gamma_{1}^{\vee}
$$

Together with the filtrations $W_{-6}=W_{-5}=W_{-4}=\langle[p]\rangle \subset W_{-3}=H_{\text {even }}$, and $\langle[p]\rangle=\mathcal{F}^{-3} \cap M_{-6}, \quad\left\langle\left[\mathbb{P}^{1}\right]\right\rangle=\mathcal{F}^{-2} \cap M_{-4}, \quad\left\langle\left[\mathbb{P}^{2}\right]\right\rangle=\mathcal{F}^{-1} \cap M_{-2}, \quad$ this determines the A-model (relative) variation matching that on the B-model.

Finally, consider the formal quantum product

$$
\begin{aligned}
& e_{1}^{\vee} * e_{3}^{\vee}=0, \quad e_{1}^{\vee} * e_{2}^{\vee}=-3 e_{3}^{\vee}, \quad e_{1}^{\vee} * e_{1}^{\vee}=-3 \Phi^{\prime \prime} e_{2}^{\vee}, \\
& e_{2}^{\vee} * e_{3}^{\vee}=0, \quad e_{3}^{\vee} * e_{3}^{\vee}=0, \quad e_{2}^{\vee} * e_{2}^{\vee}=0,
\end{aligned}
$$

where we continue to identify classes under $\overline{\mathrm{mir}}$. This is compatible with the ordinary cup product in the sense that

$$
\begin{aligned}
& e_{1}^{\vee} \cup e_{3}^{\vee}=\left[\mathbb{P}^{2}\right] \cup[p]=0, \\
& e_{1}^{\vee} \cup e_{2}^{\vee}=\left[\mathbb{P}^{2}\right] \cup\left[\mathbb{P}^{1}\right]=\left(\mathbb{P}^{2} \cdot \mathbb{P}^{1}\right)_{Y \circ}[p]=-3[p]=-3 e_{3}^{\vee}, \quad \text { and } \\
& e_{1}^{\vee} \cup e_{1}^{\vee}=\left[\mathbb{P}^{2}\right] \cup\left[\mathbb{P}^{2}\right]=-3\left[\mathbb{P}^{1}\right]=-3 e_{2}^{\vee},
\end{aligned}
$$

the last of which contains the leading term of $-3 \Phi^{\prime \prime}=-3+\cdots$.

Proposition 2.1. With the product (2.5), (2.3) may be rewritten

$$
\nabla=d+\left(\frac{1}{3} e_{1}^{\vee}\right) \otimes \frac{d Q}{2 \pi \sqrt{-1} Q}
$$

in terms of the quantum product with the zero-section $\mathbb{P}^{2} \subset K_{\mathbb{P}^{2}}$.

This motivates the following

Problem 2.2. Develop a general theory of quantum cohomology for the local setting recovering $W_{\bullet}$ and $\nabla$ on $H_{\text {even }}\left(Y^{\circ}\right)$ as above. In particular, this 
will entail the construction of dual admissible PVMHS on $H^{\text {even }}$ and $H_{\text {even }}$ for the total space of the canonical line bundle over a toric Fano orbifold.

We will obtain a solution for our running example in the next section. Remark that the required $\nabla$ is defined in passing in [17, Section 2.5], which we shall comment in below (see Remark 3.2).

The Abel-Jacobi maps from [10] touched on above may be viewed as maps from $K_{2}^{\mathrm{alg}}(W)=K_{2}(\operatorname{Coh}(W))$ to $(\mathbb{C} / \mathbb{Z}(2)$-valued $)$ functionals on (classes of) Lagrangian 1-cycles on $W$. Noting that $W^{\circ}$ is also an elliptic curve, we propose

Problem 2.3. Derive (in general) a homological mirror to $A J$. This would produce a "symplectic regulator" map from $K_{2}\left(\operatorname{Fuk}\left(W^{\circ}\right)\right)$ to functionals on coherent sheaves on $W^{\circ}$. The functional mirroring the $A J$ class in our example would send $\mathcal{O}_{p} \mapsto \frac{(2 \pi \sqrt{-1})^{2}}{3} \mathcal{T}$ and $\mathcal{O}_{W}$ 。

The motivation for such a quantum $A J$ map is clear: it would bring Beilinson's conjectures directly to bear upon the arithmetic of GromovWitten (GW) invariants, in the context of the A-model VHS on quantum cohomology. A first step might be to construct, in our example, a mirror in $K_{2}\left(\operatorname{Fuk}\left(W^{\circ}\right)\right)$ to the toric symbol $\{x, y\} \in K_{2}^{\text {alg }}(W)$ (i.e., the higher cycle), by representing $K_{2}^{\mathrm{alg}}(W)$ using the Quillen category of $\operatorname{Coh}(W)$ and applying homological mirror symmetry for elliptic curves.

\section{Closed to local}

We begin by summarizing a computation from [8]. The setting is a 2parameter family $X_{\xi_{1}, \xi_{2}}$ of $h^{2,1}=2 \mathrm{CY} 3$-folds over a product $\left(\Delta^{*}\right)^{2}$ of punctured disks, with $\hat{\eta} \in \Omega^{3}(X)$. The mirror $\left(h^{1,1}=2\right)$ CY has an elliptic fibration

$$
X^{\circ} \stackrel{\bar{\rho}}{\rightarrow} \mathbb{P}^{2}
$$

with

- zero-section $D_{2} \cong \mathbb{P}^{2}$,

- a line $C_{2} \cong \mathbb{P}^{1} \subset D_{2}$ with preimage $D_{1}=\bar{\rho}^{-1}\left(C_{2}\right)$ and

- a fiber $C_{1}=\bar{\rho}^{-1}(p)$. 
We will use the bases

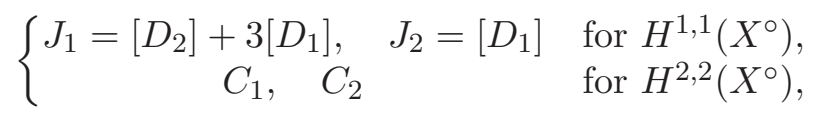

which are dual under cup product. The period vector for $\hat{\eta}$ takes the form

$$
\left(\Pi_{0}, \tau_{1} \Pi_{0}, \tau_{2} \Pi_{0}, \partial_{\tau_{1}} \tilde{\Phi}, \partial_{\tau_{2}} \tilde{\Phi}, 2 \tilde{\Phi}-\delta_{\tau_{1}} \tilde{\Phi}-\delta_{\tau_{2}} \tilde{\Phi}\right)
$$

where $\Pi_{0}$ is the "holomorphic period" and

$$
\tilde{\Phi}:=\frac{3}{2} \tau_{1}^{3}+\frac{3}{2} \tau_{1}^{2} \tau_{2}+\frac{1}{2} \tau_{1} \tau_{2}^{2}+\left\{\frac{17}{4} \tau_{1}+\frac{3}{2} \tau_{2}+C\right\}+\frac{1}{(2 \pi \sqrt{-1})^{3}} \sum_{d_{1}, d_{2}} \tilde{N}_{d_{1}, d_{2}} q_{1}^{d_{1}} q_{2}^{d_{2}}
$$

is the prepotential. ${ }^{8}$ Here, $q_{j}=e^{2 \pi \sqrt{-1} \tau_{j}}(j=1,2)$ are the disk-coordinates ${ }^{9}$ and $\tilde{N}_{d_{1}, d_{2}}$ is the GW invariant of the class $d_{1}\left[C_{1}\right]+d_{2}\left[C_{2}\right]$ on $X^{\circ}$; the Kähler class is simply $\tau_{1} J_{1}+\tau_{2} J_{2}$.

Now we take $\tau_{1} \rightarrow i \infty\left(q_{1} \rightarrow 0\right)$ considered as the "large volume limit" for the fibers of $\bar{\rho}$. For the purposes of GW theory on the A-model, in this limit $X^{\circ}$ is equivalent to the total space of $\mathcal{N}_{D_{2} / X^{\circ}} \cong K_{\mathbb{P}^{2}}$, i.e., $Y^{\circ}$ in the last section (with the map $\rho: Y^{\circ} \rightarrow \mathbb{P}^{2}$ ). On the B-model, which we shall henceforth ignore, the periods remaining finite are $\Pi_{0}, \tau_{2} \Pi_{0}$, and

$$
\left(\partial_{\tau_{1}}-3 \partial_{\tau_{2}}\right) \tilde{\Phi}=\frac{1}{2} \tau_{2}^{2}-\frac{1}{4}+\frac{1}{(2 \pi \sqrt{-1})^{2}} \sum_{d_{1}, d_{2}} \tilde{N}_{d_{1}, d_{2}}\left(d_{1}-3 d_{2}\right) q_{1}^{d_{1}} q_{2}^{d_{2}}
$$

Indeed, actually taking the limit of (3.1) (and writing $\mathcal{T}:=\tau_{2}, Q:=$ $\left.e^{2 \pi \sqrt{-1} \mathcal{T}}, N_{d}:=\tilde{N}_{0, d}\right)$ defines the local prepotential

$$
\Phi_{\text {loc }}:=\frac{1}{2} \mathcal{T}^{2}-\frac{1}{4}-\frac{1}{(2 \pi \sqrt{-1})^{2}} \sum_{d} 3 d N_{d} Q^{d}
$$

in agreement with $(2.2) \cdot{ }^{10}$

The next step is to consider the limit of the quantum products of classes in $H^{\text {even }}\left(X^{\circ}\right)$ which come from $H_{c}^{\text {even }}\left(Y^{\circ}\right)\left(\cong H_{\text {even }}\left(Y^{\circ}\right)\right)$, namely $[p],\left[C_{2}\right]$,

\footnotetext{
${ }^{8}$ This is the usual GW prepotential plus the bracketed lower-order correction terms.

${ }^{9}$ That is, the mirror map sends $\left(\xi_{1}, \xi_{2}\right) \mapsto\left(q_{1}, q_{2}\right)$ [op. cit., Section 6.1] and we may replace the former by the latter as coordinates on $\Delta^{2}$.

${ }^{10}$ In fact, by a computation in [14], $\Phi=\Phi_{\text {loc }}-\frac{1}{2} \mathcal{T}+\frac{1}{2}$.
} 
and

$$
\left[D_{2}\right]=J_{1}-3 J_{2}
$$

In general, the only interesting products (not given by the cup product) are

$$
J_{j} * J_{k}=\sum_{\ell}\left(\partial_{\tau_{j}} \partial_{\tau_{k}} \partial_{\tau_{\ell}} \tilde{\Phi}\right)\left[C_{\ell}\right] .
$$

So (using (3.1)) we have

$$
\begin{aligned}
{\left[D_{2}\right] *\left[D_{2}\right] } & =\left(\partial_{\tau_{1}}-3 \partial_{\tau_{2}}\right)^{2}\left(\partial_{\tau_{1}} \tilde{\Phi}\left[C_{1}\right]+\partial_{\tau_{2}} \tilde{\Phi}\left[C_{2}\right]\right) \\
& =-3\left[C_{2}\right]+\sum_{d_{1}, d_{2}} \tilde{N}_{d_{1}, d_{2}}\left(d_{1}-3 d_{2}\right)^{2}\left(d_{1}\left[C_{1}\right]+d_{2}\left[C_{2}\right]\right) q_{1}^{d_{1}} q_{2}^{d_{2}}
\end{aligned}
$$

whereupon taking the limit

$$
\begin{aligned}
\lim _{q_{1} \rightarrow 0}\left[D_{2}\right] *\left[D_{2}\right] & =\left\{-3+\sum_{d} N_{d}(-3 d)^{2} d Q^{d}\right\}\left[C_{2}\right] \\
& =-3\left\{1-\sum_{d} 3 d^{3} N_{d} Q^{d}\right\}\left[C_{2}\right]
\end{aligned}
$$

gives $\left[\mathbb{P}^{2}\right] *\left[\mathbb{P}^{2}\right]=-3 \Phi^{\prime \prime}\left[\mathbb{P}^{1}\right]$, which is exactly what we wanted.

This makes a case for the general principle that the "local restriction" of the quantum product in a closed CY should remain finite under an appropriate large volume limit. Beyond establishing this, a solution to Problem 2.2 would have to show the result is consistent with a formula of the shape ${ }^{11}$

$$
\alpha *_{\operatorname{loc}} \beta:=\sum_{k} \sum_{d}\left\langle\alpha, \beta, \phi^{k}\right\rangle_{0,3, d}^{\mathrm{loc}} \phi_{k} e^{\langle d, \mathcal{T}\rangle}
$$

for $\alpha, \beta \in H_{\text {even }} \cong H_{c}^{\text {even }}, \mathcal{T} \in H^{2}, d \in H_{2}$, and $\phi^{k}$ resp. $\phi_{k}$ dual bases of $H^{\text {even }}$ resp. $H_{\text {even }}$.

The resulting local quantum cohomology would then provide a direct A-model approach to "most" of the variation of mixed Hodge structure (the $\left\{I^{p, q}\right\}$ and $\nabla$-flat structure), leaving only the

\footnotetext{
${ }^{11}$ For $Y^{\circ} \cong K_{\mathbb{P}} \rightarrow \mathbb{P}$ with $\mathbb{P}$ a toric Fano surface, negativity of $K_{\mathbb{P}}$ allows us to express the local invariants as closed invariants $\left\langle\alpha, \beta, \phi^{k}\right\rangle_{0,3, \iota_{*}(d)}$ for $\overline{Y^{\circ}}:=\mathbb{P}(\mathcal{O} \oplus$ $\left.K_{\mathbb{P}}\right) \stackrel{\iota}{\supset} Y^{\circ}$; cf. [6, Section 9].
} 
PRoblem 3.1. Extend Iritani's construction of an integral structure on $\nabla$ flat sections of $H_{\text {even }}\left(Y^{\circ}\right)$ to the local CY setting (with $Y^{\circ}=K_{\mathbb{P}_{\Delta}}$ ).

This is easily accomplished in our running example by "taking LMHS along $q_{1}=0$ " of the $\mathbb{Z}$-VHS over $\left(\Delta^{*}\right)^{2}$ (common to both the A- and Bmodels). More precisely, if $T_{1}$ denotes the monodromy about $q_{1}=0$, with logarithm $N_{1}$, then the limiting variation of MHS takes the form

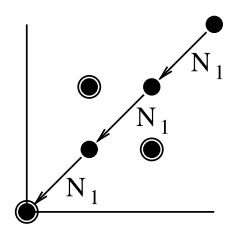

where the circled bullets denote $\operatorname{ker}\left(N_{1}\right)=\operatorname{ker}\left(T_{1}-\mathrm{id}\right)$. For our purposes, it will suffice to compute the limit of the $T_{1}$-invariant "cycles" in the $\hat{\Gamma}$-integral structure on the closed A-model VHS $H^{\text {even }}\left(X^{\circ}\right)$.

Indeed, together with the Clemens-Schmid sequence, the assumption that " $Y^{\circ}$ is the A-model limit of $X^{\circ}$ " implies that

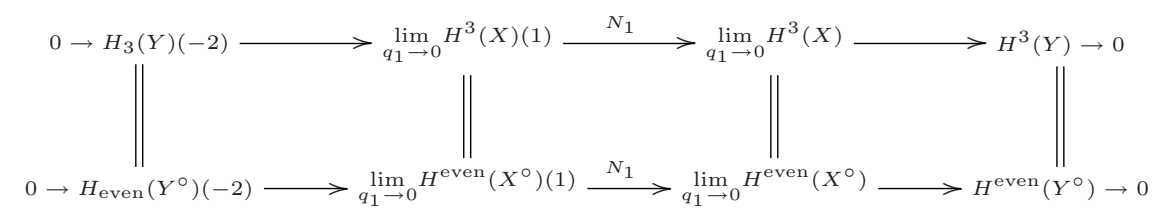

is an exact sequence of VMHS (in $q_{2}$ ). Iritani's procedure necessarily gives integral $\nabla$-flat sections $\left\{\hat{\gamma}_{i}\right\}_{i=1}^{6}$ in $H^{\text {even }}\left(X^{\circ}\right)$, with $\left\{\hat{\gamma}_{4}, \hat{\gamma}_{5}, \hat{\gamma}_{6}\right\} \subset \operatorname{im}\left(N_{1}\right)$ and $\left\{\hat{\gamma}_{1}^{\mathrm{V}}, \hat{\gamma}_{2}^{\mathrm{V}}, \hat{\gamma}_{3}^{\mathrm{V}}\right\} \subset \operatorname{ker}\left(N_{1}\right)$, such that ${ }^{12}$

$$
\left[X^{\circ}\right]\left(=\frac{\hat{\eta}}{\Pi_{0}}\right)=\hat{\gamma}_{1}+\tau_{2} \hat{\gamma}_{2}+\left\{\left(\partial_{\tau_{1}}-3 \partial_{\tau_{2}}\right) \tilde{\Phi}\right\} \hat{\gamma}_{3}+\sum_{j=4}^{6} \hat{\pi}_{j}(\underline{\tau}) \hat{\gamma}_{j} .
$$

\footnotetext{
${ }^{12}$ Here the $\left\{\pi_{j}(\underline{\tau})\right\}$ are defined by the equation, and the $\left\{\hat{\gamma}_{i}\right\}$ exist by virtue of the agreement between $\gamma\left(K_{0}^{\text {num }}\left(X^{\circ}\right)\right)$ (cf. (1.5)) and the B-model $\mathbb{Z}$-structure. (The authors do not know explicit $\xi_{i} \in K_{0}^{\text {num }}\left(X^{\circ}\right)$ with $\gamma\left(\xi_{i}\right)=\hat{\gamma}_{i}$; whereas by [15] these must exist, the argument here uses only what we know from the B-model, and so does not require them.)
} 
Taking the limit whilst killing $\operatorname{im}\left(N_{1}\right)$, then making the change of basis ${ }^{13}$ $\left\{\hat{\gamma}_{1}, \hat{\gamma}_{2}, \hat{\gamma}_{3}\right\}=:\left\{\gamma_{1}+\frac{1}{2} \gamma_{3}, \gamma_{2}-\frac{1}{2} \gamma_{3}, \gamma_{3}\right\}$, recovers

$$
e_{3}=\gamma_{1}+\mathcal{T} \gamma_{2}+\Phi \gamma_{3}
$$

in $H^{\text {even }}\left(Y^{\circ}\right)$.

Of course, in analogy to (3.2), it would be better to solve Problem 3.1 in a manner intrinsic to the local A-model. That is, there should be a direct construction as in (1.5) assigning flat sections of $H_{\text {even }}\left(Y^{\circ}\right)$ to classes in $K_{0}^{c}\left(Y^{\circ}\right)$, and "compatible with monodromy". In our example, (2.4) has this compatibility, since $\otimes \mathcal{O}_{Y^{\circ}}\left(-J_{2}\right)$ on the coherent sheaves and monodromy about $q=0$ on the cycles have the same matrix

$$
\left(\begin{array}{lll}
1 & 1 & 0 \\
0 & 1 & 1 \\
0 & 0 & 1
\end{array}\right) .
$$

Remark 3.2. As mentioned above, Iritani [15, Section 2.5] briefly describes an extension of the Dubrovin connection and $\hat{\Gamma}-\mathbb{Z}$-structure which should provide such a "direct" solution to Problem 3.1. Whether this produces an admissible $\mathbb{Z}$-PVMHS, or (more precisely) matches the VMHS on the middle cohomology of the Hori-Vafa mirror $Y$, remains to be verified. It would, for example, suffice to exhibit a degenerating family of CY $n$-folds $X_{\xi_{1}, \xi_{2}}$ over $\Delta^{2}$ with isomorphisms (a) $H^{n}\left(X_{0, \xi}\right) \cong H_{n}\left(Y_{\xi}\right)(-n)$ and $(b) H_{\text {even }}\left(Y^{\circ}\right)(-n) \cong$ $\operatorname{ker}\left(N_{1}\right) \subseteq \lim q_{1} \rightarrow 0 H^{\text {even }}\left(X^{\circ}\right)$ of VMHS. Here (a) is a geometric/Hodgetheoretic calculation on the B-model side, while (b) is a compatibility check for the A-model $\mathbb{Z}$-structures in the compact and noncompact cases.

Apparently, either solution to Problem 3.1 still leaves us a long way from the "holy grail" of Problem 2.3.

\section{Open string}

Problem 2.3 is probably intractable without major theoretical developments. However, its rough analog in the relative situation studied by Morrison and Walcher [22] appears to be more accessible. In particular, there is nothing mysterious about the mirror of the (usual, not higher) algebraic cycle - it is just a Lagrangian.

\footnotetext{
${ }^{13}$ Note that Clemens-Schmid is only rationally exact, and that $N_{1}, N_{2}$ are only defined over $\mathbb{Q}$.
} 
The B-model in the example we consider (following [op. cit.]) comprises:

- $X=$ a double-cover of the mirror quintic family, with holomorphic form $\omega \in \Omega^{3}(X)$;

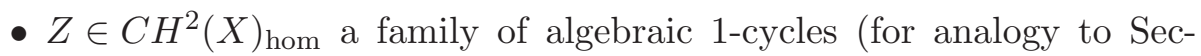
tion 2 , think " $K_{0}(\operatorname{Coh}(X))$ "); and

- $\left\langle A J_{X}^{2}(Z), \omega\right\rangle=$ the resulting "truncated normal function", solving

- the inhomogeneous Picard-Fuchs equation $D_{\mathrm{PF}}^{\omega}\left\langle A J_{X}^{2}(Z), \omega\right\rangle=: g$.

On the A-model side these data mirror to:

- $X^{\circ}=$ the Fermat quintic;

- $Z^{\circ} \cong \mathbb{R P}^{3}$ the real quintic, viewed as a Lagrangian 3-cycle (think " $K_{0}(\operatorname{Fuk}(X))$ "); and

- the open Gromov-Witten generating function whose coefficients count holomorphic disks bounding on $Z^{\circ}$,

which (under the mirror map) solves the same PF equation.

As in the closed and local stories, GW numbers are therefore obtained as power-series coefficients of a Hodge-theoretic function, with (in this latter role) the Yukawa coupling replaced by the truncated normal function.

Problem 4.1. Work out (in analogy with Sections 1 and 2) the $[\nabla]_{e}$ story. This will require the full normal function (not considered in [op. cit.]), which means computing also $\left\langle A J_{X}^{2}(Z), \nabla_{\partial_{\tau}} \omega^{3,0}\right\rangle$.

Since the B-model VMHS is an extension of the constant variation $\mathbb{Z}(-2)$ by the pure VHS $H^{3}(X)$, the extension class is defined over $\mathbb{R}$ hence given completely by $\left\langle A J_{X}^{2}(Z), \omega^{3,0}\right\rangle$ and $\left\langle A J_{X}^{2}(Z), \nabla_{\partial_{\tau}} \omega^{3,0}\right\rangle$. The extension arises geometrically from the residue exact sequence

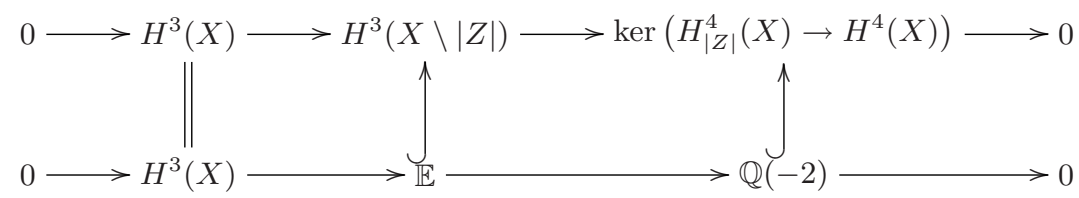

Completely missing, however, is an approach to the following. 
Problem 4.2. Can one produce the extension class from the pair $X^{\circ}, Z^{\circ}$ from the standpoint of quantum cohomology and the A-model VMHS?

To illustrate its difficulty, a naive attempt to mirror the exact sequence approach, viz.

$$
0 \rightarrow \frac{H^{\text {even }}\left(X^{\circ}\right)}{H_{Z^{\circ}}^{6}\left(X^{\circ}\right)} \rightarrow H^{\text {even }}\left(X^{\circ} \backslash Z^{\circ}\right) \rightarrow \operatorname{ker}\left(H_{Z^{\circ}}^{3}\left(X^{\circ}\right) \rightarrow H^{3}\left(X^{\circ}\right)\right) \rightarrow 0
$$

fails due to the vanishing of the third term. The result of [op. cit.], however, that the "truncated" extension class is given by the open GW generating function, gives one reason to believe the problem has interesting content.

Remark 4.3. We briefly note another interesting phenomenon that arises in the open setting, related to Remark 1.6. Even on a family of CY 3-folds defined over $\mathbb{Q}$, algebraic cycles often force an algebraic extension $L / \mathbb{Q}$ upon us, as in the case of the van Geemen lines on the mirror quintic family studied by Laporte and Walcher [19]. The resulting limits of truncated normal functions can then often be expressed in terms of the Borel regulator on $K_{3}^{\text {ind }}(L)$ (see [13] for the theoretical reason). This makes the open setting ideal terrain for exploring generalizations of the A-model $\hat{\Gamma}$-construction where the B-model LMHS does not correspond to a $\mathbb{Q}$-rational limiting motive.

\subsection{Local to open}

Recent work of Chan, Lau, Leung, Tseng and $\mathrm{Wu}[3,4,20]$ has brought to light an interesting relation between the (local) mirror map and certain open Gromov-Witten invariants for a toric Calabi-Yau manifold $Y^{\circ}$. The first three authors conjecture in [3] that the SYZ mirror construction (applied to $Y^{\circ}$ ) inverts the mirror map given by a normalized integral basis of single-logdivergent periods of the Hori-Vafa mirror $Y$. With the integrality hypothesis dropped, the conjecture is established in [4] for $Y^{\circ}=K_{Z}$ with $Z$ a compact toric Fano variety; it is known integrally for toric surfaces [20] and a handful of other examples [3], including $K_{\mathbb{P}^{2}}$.

We briefly describe the case $Y^{\circ}=K_{\mathbb{P}^{2}}$ in the notation of Section 2. Let $\ell$ be the class of a line $L\left(\cong \mathbb{P}^{1}\right)$ contained in the zero section $D\left(\cong \mathbb{P}^{2}\right) \subset Y^{\circ}$, and $\mathcal{T}\left[\rho^{-1}(L)\right] \in H^{2}\left(Y^{\circ}, \mathbb{C}\right)$ be the Kähler class with corresponding Kähler parameter $Q=e^{2 \pi i \mathcal{T}}$. Take $\beta_{0}$ to denote the class of a holomorphic disk with $\beta_{0} \cdot D=1$ bounding on a Lagrangian torus (see [3, Section 4.2.3] for the precise definition). Then the SYZ construction in [3] produces the noncompact 
Calabi-Yau in $\left(\mathbb{C}^{*}\right)^{2} \times \mathbb{C}^{2}$ given by

$$
U V=c(Q)+X+Y+\frac{Q}{X Y}
$$

where $c(Q)=1+\sum_{k \geq 1} n_{\beta_{0}+k \ell} Q^{k}$ is an open Gromov-Witten generating series. An easy change of coordinates exhibits (4.1) as the Hori-Vafa manifold $Y_{\xi}$ of $(2.1)$, with $\xi=-\frac{Q^{\frac{1}{3}}}{c(Q)}$; taking the cube gives

$$
s(Q)=-\frac{Q}{c(Q)^{3}}
$$

The observation of [op. cit.] is that (4.2) inverts the local mirror map

$$
Q(s)=e^{2 \pi \sqrt{-1} \mathcal{T}(s)}=\exp \left(\frac{1}{(2 \pi \sqrt{-1})^{2}} \int_{\mathcal{M}\left(3 \varphi_{0}\right)} \eta\right)
$$

in Section 2. So just as for $\Phi$, we have an enumerative interpretation for $\mathcal{T}$, and one can use the computation ${ }^{14}$

$$
\mathcal{T}(s)=\ell(s)+\frac{1}{2}+\frac{1}{2 \pi \sqrt{-1}} \sum_{k \geq 1}(-1)^{k} \frac{\left(\begin{array}{c}
3 k \\
k, k, k
\end{array}\right)}{k} s^{k}
$$

in [3] or [10] to compute $c(Q)=1-2 Q+5 Q^{2}-32 Q^{3}+\cdots$.

We conclude with one final

Problem 4.4. Can one use the formulae in Section 5 of [10] for the integral periods of Hori-Vafa mirrors, to establish integrality in [4]?

Remark 4.5. While this paper was under review, Problem 4.4 was answered affirmatively for $Y^{\circ}=K_{Z}$ a threefold (cf. the footnote in [2, Section 1]). We expect that the relevant computations in [10, Section 5] generalize to dimensions $n>3$ as well; the more interesting question is whether similar constructions exist for more general semi-projective toric Calabi-Yau varieties (not of the form $K_{Z}$ ).

\footnotetext{
${ }^{14}$ up to the sign and term $\frac{1}{2}$ which are required for consistency with Section 2 and $[14]$.
} 


\section{Acknowledgments}

The authors thank E. Zaslow for a helpful conversation, and the two referees for helpful suggestions. C.F.D. wishes to recognize support from the NSERC Discovery Grants Program and the second author from the NSF under Standard Grant DMS-1068974.

\section{References}

[1] P. Candelas, X. de la Ossa, P. Green and L. Parkes, A pair of manifolds as an exactly solvable superconformal theory, Nucl. Phys. B 359 (1991), 21-74.

[2] K. Chan, C.-H. Cho, S.-C. Lau and H.-H. Tseng, Gross fibrations, SYZ mirror symmetry, and open Gromov-Witten invariants for toric Calabi-Yau orbifolds, 2013, arXiv math.AG/1306.0437v3.

[3] K. Chan, S.-C. Lau and N.C. Leung, SYZ mirror symmetry for toric Calabi-Yau manifolds, J. Differential Geom. 90(2) (2012), 177-250.

[4] K. Chan, S.-C. Lau and H.-H. Tseng, Enumerative meaning of mirror maps for toric Calabi-Yau manifolds, Adv. Math. 244 (2013), 605-625.

[5] A. Chiodo, H. Iritani and Y. Ruan, Landau-Ginzburg/Calabi-Yau correspondence, global mirror symmetry and Orlov equivalence, Publ. Math. de l'IHES 119 (2014), 127-216.

[6] T. Coates and H. Iritani, On the convergence of Gromov-Witten potentials and Givental's formula, 2012, arXiv math.AG/1203.4193v1.

[7] D. Cox and S. Katz, Mirror symmetry and algebraic geometry, Math. Surveys and Monographs 68, AMS, Providence, RI, 1999.

[8] T.-M. Chiang, A. Klemm, S.-T. Yau and E. Zaslow, Local mirror symmetry: calculations and interpretations, ATMP 3 (1999), 495-565.

[9] P. Deligne, Local behavior of Hodge structures at infinity, in Mirror Symmetry II, eds. B. Green, S.-T. Yau, AMS/IP Stud. Adv. Math., American Mathematical Society, Providence, RI, 1997, 683-699.

[10] C. Doran and M. Kerr, Algebraic K-theory of toric hypersurfaces, CNTP 5(2) (2011), 397-600.

[11] C. Doran and J. Morgan, Mirror symmetry and integral variations of Hodge structure underlying one-paramameter families of Calabi-Yau 
threefolds, in 'Mirror Symmetry V', 38, AMS/IP Stud. Adv. Math., 2006, 517-537.

[12] M. Green, P. Griffiths and M. Kerr, Neron models and boundary components for degenerations of Hodge structures of mirror quintic type, in 'Curves and Abelian Varieties', ed. V. Alexeev, 465, Contemp. Math 2007, AMS, 71-145.

[13] M. Green, P. Griffiths and M. Kerr, Neron models and limits of AbelJacobi mappings, Compos. Math. 146 (2010), 288-366.

[14] S. Hosono, Central charges, symplectic forms, and hypergeometric series in local mirror symmetry, in 'Mirror Symmetry V', eds. Lewis, Yau, Yui, AMS/IP Stud. Adv. Math. 38, 2006, 405-440

[15] H. Iritani, An integral structure in quantum cohomology and mirror symmetry for toric orbifolds, Adv. Math. 222(3) (2009), 10161079 .

[16] H. Iritani, Quantum cohomology and periods, Ann. Inst. Fourier 61 (2011), 2909-2958.

[17] H. Iritani, Ruan's conjecture and integral structures in quantum cohomology, Adv. Stud. Pure Math. 59 (2010), 111-166.

[18] M. Kontsevich, L. Katzarkov and T. Pantev, Hodge theoretic aspects of mirror symmetry, from Hodge theory to integrability and TQFT tt*geometry, Proc. Symp. Pure Mathematics, 78, Amer. Math. Soc., Providence, RI, 2008, 87-174.

[19] G. Laporte and J. Walcher, Monodromy of an inhomogeneous PicardFuchs equation, SIGMA 8 (2012), 056, 10 pp.

[20] S.-C. Lau, N.C. Leung and B. Wu, Mirror maps equal SYZ maps for toric Calabi-Yau surfaces, Bull. Lond. Math. Soc. 44 (2012), 255-270.

[21] K. Mohri, Y. Onjo and S.-K. Yang, Closed sub-monodromy problems, local mirror symmetry and branes on orbifolds, Rev. Math. Phys. 13 (2001), 675-715.

[22] D. Morrison and J. Walcher, D-branes and normal functions, Adv. Theor. Math. Phys. 13(2) (2009), 553-598.

[23] G. Pearlstein, Variations of mixed Hodge structure, Higgs fields, and quantum cohomology, Manuscripta Math. 102(3) (2000), 269-310. 
Department of Mathematical and Statistical Sciences

University of Alberta

CANADA

E-mail address: charles.doran@ualberta.ca

Department of Mathematics, Campus Box 1146

WASHINGTON UNIVERSITY IN ST. LOUIS

St. Louis, MO 63130

USA

E-mail address: matkerr@math. wustl.edu

Received August 2, 2013 
